\title{
UNCERTAINTY MODEL AND SINGULARITIES OF 3-2-1 WIRE-BASED TRACKING SYSTEMS
}

\author{
Federico Thomas**, Erika Ottaviano*, Lluís Ros**, Marco Ceccarelli* \\ *Laboratorio di Robotica e Meccatronica \\ Università degli Studi di Cassino, Via G. Di Biasio, 43, 03043 Cassino, Italy \\ \{ootaviano, ceccarelli\}@unicas.it \\ ** Institut de Robòtica i Informàtica Industrial (CSIC-UPC) \\ Llorens Artigas 4-6, 08028 Barcelona, Spain \\ $\{$ IIros, fthomas\}@iri.upc.es
}

\begin{abstract}
This paper presents an ellipsoidal set-membership uncertainty model for wire-based tracking systems with wire length uncertainties and joint clearances. Although the proposed model is valid for any number of wires and configurations, including singularities, it has been particularized to a 3-2-1 parallel wire mechanism. The Euler's tetrahedron formula has been used to obtain a numerically stable solution to the direct kinematics of this particular tracking system as well as a compact characterization of its singularities directly expressed in terms of the wire lengths. It is also shown that these singularities, when expressed in the configuration space of the moving element, can be identified as C-surfaces associated with the three basic contacts between polyhedra, which have been widely studied in the context of path planning.
\end{abstract}

Keywords: Tracking systems, uncertainty manipulation, kinematic singularities. Euler's tetrahedron formula.

\section{Introduction}

Many systems for measuring position and orientation of moving objects, also known as tracking systems, have been developed. They can be classified according to the measuring principle and used technology (Hightower and Borriello, 2001). Most of the instruments such as cameras, theodolites, laser and wire systems use triangulation or trilateration techniques. Trilateration and triangulation determine the relative position between points by using the geometry of triangles. Triangulation uses measurements of both distances and angles, whereas trilateration uses only distance measurements. 
Measurement systems can be also classified according to their characteristics, such as accuracy, resolution, cost, measurement range, portability, and calibration procedure. Laser tracking systems exhibit good accuracy, which can be less than $1 \mu \mathrm{m}$ if the system is well calibrated. Unfortunately, they are very expensive, their calibration procedure is time consuming, and they are sensitive to the environment. Vision systems have an accuracy of $0.1 \mathrm{~mm}$, they are low cost portable devices but their calibration procedure can be complicate. Wire systems have an accuracy of $0.1 \mathrm{~mm}$, they are also low cost portable devices but capable of measuring large displacements. Moreover, they exhibit a good compromise among accuracy, measurement range, cost and operability.

Wire-based tracking systems consist of a fixed base and a platform connected by six wires whose tension is maintained, while the platform is moved, by pulleys and spiral springs on the base. They can be modelled as six-degree-of-freedom parallel manipulators because wires can be seen as extensible legs connecting the platform and the base by means of spherical and universal joints, respectively. One of these wire mechanisms was used in (Jeong et al., 1999) for robot pose, i.e. position and orientation, measurements. The proposed system was studied as a 2-22 Gough-Stewart platform and, as a consequence, its direct kinematics could only be solved by a numerical method. This clearly limited its use in real-time measurements. Alternatively, (Geng, 1994) and (Ceccarelli et al, 1999) used a wire configuration equivalent to a 3-2-1 GoughSteward platform whose direct kinematics can be solved in closed-form by using trilateration.

Dimension deviations due to fabrication tolerances, wire length uncertainties, and joint clearances may result in an unacceptable performance of wire-based tracking systems. Wire length uncertainties are due, among other factors, to deflections caused by wire self-weights and to discretization errors in the transducers. A way to compensate the self-weight deflections is presented in (Jeong et al., 1999). Joint clearances are due to the uncertainty related to the position of the wires with respect to the corresponding joint holes on the base. They become more evident with time because of the abrasion of the hole due to wire friction. Unlike the effect of the dimension deviations, the effect of wire length uncertainties and joint clearances can not be eliminated by calibration because of their random nature. How these two factors contribute to the measured pose error and how this error evolves when the system approaches a kinematic singularity are the two issues addressed in this paper.

Most error analysis techniques assume that the data are corrupted by random noise whose probability density function is usually assumed to 
be gaussian, as in (Zhu and Ting, 2000). Real-world uncertainties, however, also include nongaussian, nonwhite noise and systematic errors. These uncertainties can easily be considered in a set theoretic setting which consists in defining bounds for the uncertain variables. The main problem with this kind of uncertainty description is that, although the initial uncertainty sets have simple shapes, the result of principal operations with them have a complicated shape. This is why some canonical sets, that depend on a fixed number of parameters, are introduced for the approximation of uncertainty sets. Among many possibilities, ellipsoids are usually taken as these canonical sets because they: (a) can be concisely described; (b) provide a satisfactory approximation of convex sets in most applications; (c) can be represented using matrices interpretable as weighted covariance matrices; and (d) are invariant, as a class, under affine transformations.

Most ellipsoidal calculus are unable to deal with degenerate cases, that is, with situations in with ellipsoids extend to infinity in some directions. Here this is an important issue because this is what happens when a tracking system approaches a kinematic singularity, as it will be shown later. Fortunately, the calculus presented in (Ros et al., 2002) is immune to degeneracies and can readily applied here.

This paper is organized as follows. Section 2 presents the adopted uncertainty model and Section 3 gives a description of the singularities first in terms of the wire lengths and then in terms of the configuration of the moving platform. Finally, Section 4 gives the conclusions.

\section{The uncertainty model}

In order to describe the relative position between base and platform, let us introduce an absolute and a relative frame fixed to the base and the platform, respectively, as shown in Figure 2a. The pose of the platform with reference to the base is given by the configuration vector $\mathbf{q}=(\boldsymbol{\Omega}, \mathbf{t})$, where $\mathbf{t}$ stands for the translation vector and $\boldsymbol{\Omega}$ for the orientation vector given in Euler's angles, i.e. $\mathbf{q} \in \Re^{3} \times S O(3)$. The corresponding rotation matrix will be noted $\mathbf{R}(\boldsymbol{\Omega})$. The unit vector along wire $i$ will be noted $\mathbf{e}_{i}$ and $l_{i}$ the corresponding wire length. The centers of the articulations on the base and the platform for wire $i$ will be denoted $\mathbf{a}_{i}$ and $\mathbf{b}_{i}$, respectively, as shown in Figure 2a. According to these definitions $\mathbf{R}(\boldsymbol{\Omega}) \mathbf{b}_{i}+\mathbf{t}=\mathbf{a}_{i}+l_{i} \mathbf{e}_{i}$. Thus, the wire length $l_{i}$ can be expressed as:

$$
l_{i}=\left\|\mathbf{R}(\boldsymbol{\Omega}) \mathbf{b}_{i}+\mathbf{t}-\mathbf{a}_{i}\right\| .
$$

Then, its linearization around the nominal values of $\mathbf{a}_{i}$ and $\mathbf{b}_{i}\left(\mathbf{a}_{i}^{0}\right.$ and $\left.\mathbf{b}_{i}^{0}\right)$, the measured value of $l_{i}\left(l_{i}^{0}\right)$, and the calculated value of the configuration $\mathbf{q}\left(\mathbf{q}^{0}\right)$ is: 


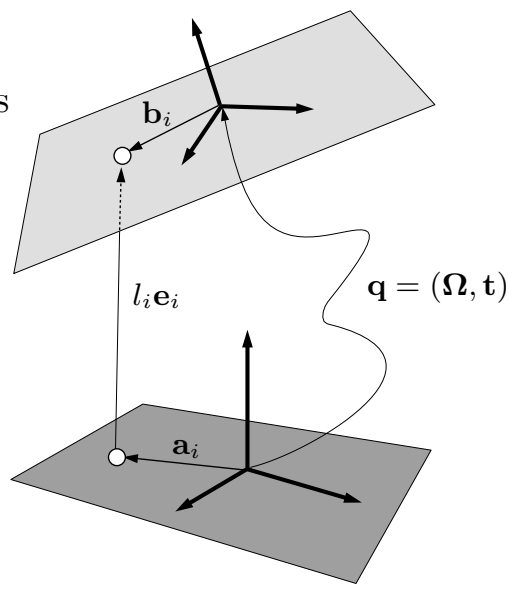

(a)

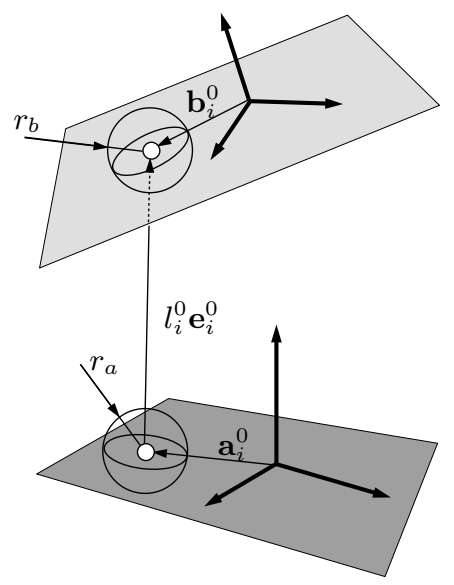

(b)

Figure 1. Magnitudes associated with wire $i(\mathbf{a})$, and its associated joint clearances (b)

$$
l_{i}-l_{i}^{0}=\frac{\partial l_{i}}{\partial \mathbf{q}}\left(\mathbf{q}-\mathbf{q}^{0}\right)+\frac{\partial l_{i}}{\partial \mathbf{a}_{i}}\left(\mathbf{a}_{i}-\mathbf{a}_{i}^{0}\right)+\frac{\partial l_{i}}{\partial \mathbf{b}_{i}}\left(\mathbf{b}_{i}-\mathbf{b}_{i}^{0}\right) .
$$

It can be proved, by taking derivatives (1), that:

$$
\frac{\partial l_{i}}{\partial \mathbf{a}_{i}}=-\mathbf{e}_{i}^{t}, \quad \frac{\partial l_{i}}{\partial \mathbf{b}_{i}}=\mathbf{e}_{i}^{t} \mathbf{R}(\boldsymbol{\Omega}), \quad \text { and } \frac{\partial l_{i}}{\partial \mathbf{q}}=\left(\begin{array}{c}
\left(\mathbf{t}-\mathbf{a}_{i}\right) \times \mathbf{e}_{i} \\
\mathbf{e}_{i}
\end{array}\right)^{t} .
$$

Substituting in (2), we get:

$$
l_{i}-l_{i}^{0}=\left(\begin{array}{c}
\left(\mathbf{t}-\mathbf{a}_{i}\right) \times \mathbf{e}_{i} \\
\mathbf{e}_{i}
\end{array}\right)^{t}\left(\mathbf{q}-\mathbf{q}^{0}\right)+v_{i}-v_{i}^{0}
$$

where $v_{i}=\mathbf{e}_{i}^{t}\left(\mathbf{R}(\boldsymbol{\Omega}) \mathbf{b}_{i}-\mathbf{a}_{i}\right)$ and $v_{i}^{0}=\mathbf{e}_{i}^{t}\left(\mathbf{R}(\boldsymbol{\Omega}) \mathbf{b}_{i}^{0}-\mathbf{a}_{i}^{0}\right)$. Finally, collecting (3) for the six wires into a single matrix expression, we get:

$$
\mathbf{J}\left(\mathbf{q}-\mathbf{q}^{0}\right)=(\mathbf{l}-\mathbf{v})-\left(\mathbf{l}^{0}-\mathbf{v}^{0}\right)
$$

where $\mathbf{J}=\left(\begin{array}{cccc}\left(\mathbf{t}-\mathbf{a}_{1}\right) \times \mathbf{e}_{1} & \left(\mathbf{t}-\mathbf{a}_{2}\right) \times \mathbf{e}_{2} & \cdots & \left(\mathbf{t}-\mathbf{a}_{6}\right) \times \mathbf{e}_{6} \\ \mathbf{e}_{1} & \mathbf{e}_{2} & \cdots & \mathbf{e}_{6}\end{array}\right)^{t}$, $\mathbf{l}=\left(l_{1}, \ldots, l_{6}\right)$, and $\mathbf{v}=\left(v_{1}, \ldots, v_{6}\right)$.

Note that the above formulation is valid for any wire configuration and can be extended to any number of wires. Note also that the rows of $\mathbf{J}$ are the Plücker coordinates of an arrangement of lines equivalent to that of the wire lines. Actually, $\mathbf{J}$ is the linear mapping between 
wire extensions and platform velocities, i.e. the inverse Jacobian of the associated parallel mechanism.

Let us assume that the real value of $\mathbf{a}_{i}$ is inside a sphere of radius $r_{a}$ centered around $\mathbf{a}_{i}^{0}$ as shown in Figure $2 \mathrm{~b}$. The same can be done for the platform joint clearances assuming that the radii of the corresponding spheres are $r_{b}$. Then, it can be checked that

$$
\left(\mathbf{v}-\mathbf{v}^{0}\right)^{t}\left(\mathbf{v}-\mathbf{v}^{0}\right) \leq 6\left(r_{a}+r_{b}\right)^{2}
$$

is a conservative bound for $\mathbf{v}$. We can also assume that all wire lengths have the same error bounds so that

$$
\left(\mathbf{l}-\mathbf{l}^{0}\right)^{t}\left(\mathbf{l}-\mathbf{l}^{0}\right) \leq 6 r_{l}^{2}
$$

also provides a conservative bound for $\mathbf{l}$. Then, the Minkowski difference between the spheres (6) and (5), that is

$$
\left((\mathbf{l}-\mathbf{v})-\left(\mathbf{l}^{0}-\mathbf{v}^{0}\right)\right)^{t}\left((\mathbf{l}-\mathbf{v})-\left(\mathbf{l}^{0}-\mathbf{v}^{0}\right)\right) \leq 6\left(r_{a}+r_{b}+r_{l}\right)^{2},
$$

gives us the uncertainty region for $(\mathbf{l}-\mathbf{v})$ (Ros et al., 2002).

Equation (7), together with (4), leads to:

$$
\left(\mathbf{q}-\mathbf{q}^{0}\right)^{t} \mathbf{J}^{t} \mathbf{J}\left(\mathbf{q}-\mathbf{q}^{0}\right) \leq 6\left(r_{a}+r_{b}+r_{l}\right)^{2},
$$

which is an ellipsoidal bound for the uncertainty region associated with $\mathbf{q}$, i.e. the configuration of the moving platform. Since $\mathbf{J}$ is a square matrix, it can be shown that the volume of the region defined by (8) is proportional to the inverse of $\operatorname{det}(\mathbf{J})$. In other words, the uncertainty region using this model is unbounded if, and only if, $\mathbf{J}$ is singular. The next section is devoted to the characterization of these singularities.

\section{Singular configurations}

Since the rows of $\mathbf{J}$ are the Plücker coordinates of lines, it is possible to characterize the singularities in terms of the geometry of linearly dependent sets of lines (Pottmann and Wallner, 2001). The set of conditions is typical for many parallel manipulators (McCarthy, 2000). Nevertheless, herein we will use an ad hoc approach that takes advantage of the 3-2-1 wire configuration.

First of all, let us define the function

$$
\Xi\left(\mathbf{p}_{1}, \mathbf{p}_{2}, \mathbf{p}_{3}, \mathbf{p}_{4}\right)=\left|\begin{array}{ccccc}
0 & r_{12} & r_{13} & r_{14} & 1 \\
r_{21} & 0 & r_{23} & r_{24} & 1 \\
r_{31} & r_{32} & 0 & r_{34} & 1 \\
r_{41} & r_{42} & r_{43} & 0 & 1 \\
1 & 1 & 1 & 1 & 0
\end{array}\right|,
$$




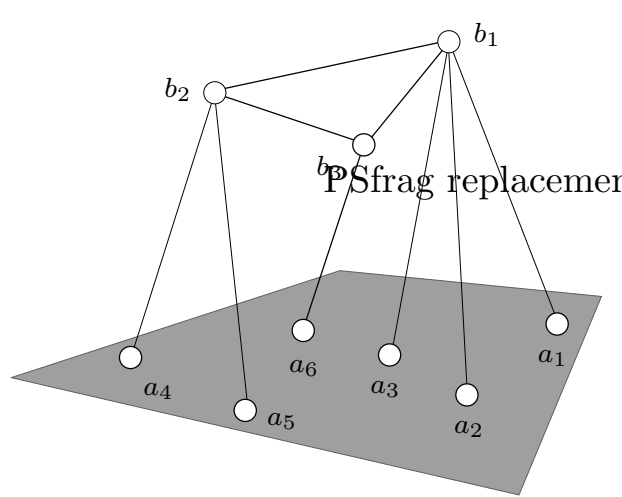

(a)

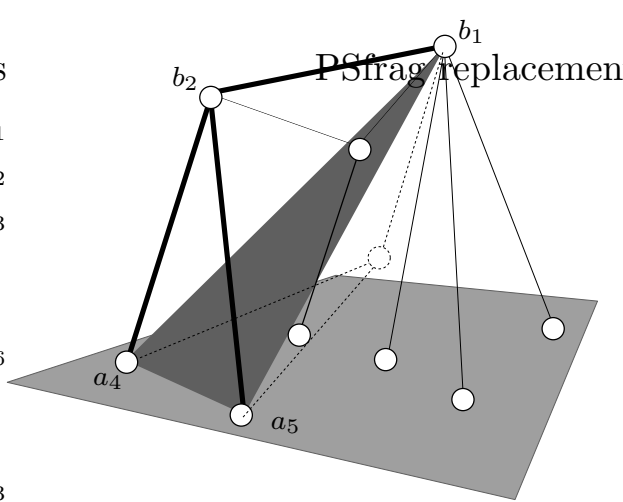

(c)

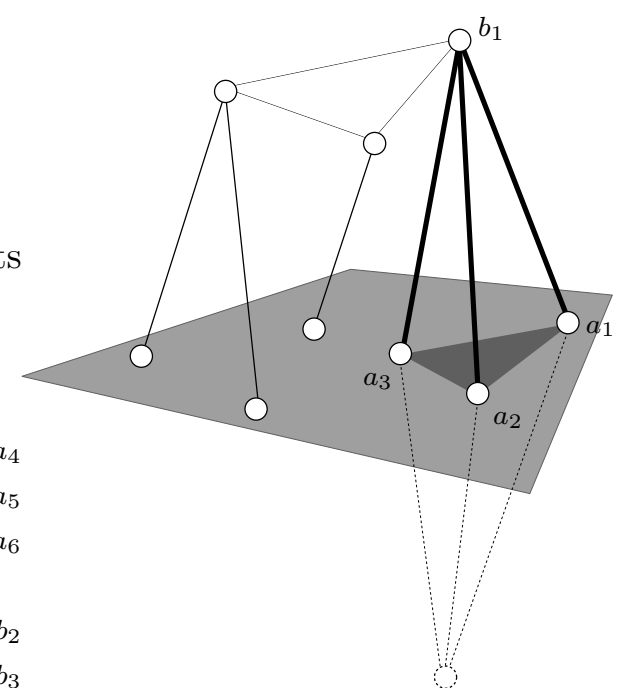

(b)

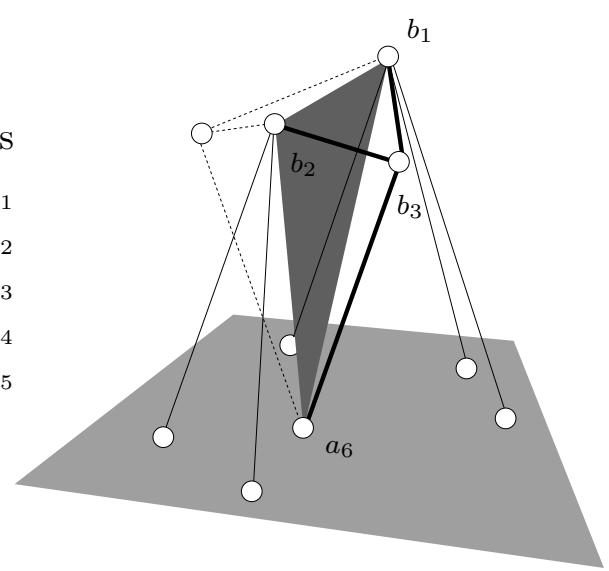

(d)

Figure 2. General kinematic model of a 3-2-1 tracking system (a). There are up to 8 possible solutions for the configuration of the platform compatible with a set of wire lengths due to the two possible solutions for the location of $\mathbf{b}_{1}(\mathbf{b}), \mathbf{b}_{2}(\mathbf{c})$, and $\mathbf{b}_{3}(\mathbf{d})$. 
where $\mathbf{p}_{1}, \ldots, \mathbf{p}_{4}$ are four points in $\Re^{3}$ and $r_{i j}$ is the square of the distance between $\mathbf{p}_{i}$ and $\mathbf{p}_{j}$. It can be shown that the volume, $V$, of the tetrahedron defined by these four points is related to (9) through the formula $288 V^{2}=\Xi\left(\mathbf{p}_{1}, \mathbf{p}_{2}, \mathbf{p}_{3}, \mathbf{p}_{4}\right)$. If determinant (9) vanishes, $\mathbf{p}_{1}, \mathbf{p}_{2}, \mathbf{p}_{3}$, and $\mathbf{p}_{4}$ lie on the same plane. If it gives a negative value, the tetrahedron cannot be assembled with the given distances. Although it is generally accepted that this remarkable formula derives from a development given by Euler in 1753 (Dörrie, 1965), the Italian painter Piero della Francesca already obtained an equivalent result around 1490 (Peterson, 1997).

Formula (9) has a 2D counterpart (known as Herron's formula) that allows us to obtain the area of a triangle in terms of the lengths of its edges. As a consequence, any of the heights of a tetrahedron can readily be obtained by dividing its volume by the corresponding base area using these formulas. The important point here is that (9) and its 2D counterpart are numerically stable and coordinate-free. Hence, this permits to solve the direct kinematics of a 3-2-1 tracking mechanism in an alternative way to that presented in (Ceccarelli et al, 1999), as explained bellow.

In a 3-2-1 wire-based tracking system, some of the joints in the platform coincide in the way shown in Figure 2a. This configuration greatly simplifies the analysis of the direct kinematics of the system. Indeed, given $l_{1}, l_{2}$, and $l_{3}$, the location of $\mathbf{b}_{1}$ can be obtained using the volume and area formulas given above. Note that there are two possible solutions which are specular with respect to the plane defined by $\mathbf{a}_{1}, \mathbf{a}_{2}$, and $\mathbf{a}_{3}$ (Figure $2 b$ ). Once one of these two solutions for $\mathbf{b}_{1}$ is chosen, $\mathbf{a}_{4}, \mathbf{a}_{5}, \mathbf{b}_{1}$ and $\mathbf{b}_{2}$ define another tetrahedron with known edge lengths. Again, there are two possible specular locations for $\mathbf{b}_{2}$, in this case with respect to the plane defined by $\mathbf{a}_{4}, \mathbf{a}_{5}$, and $\mathbf{b}_{1}$ (Figure 2c). Finally, after choosing one of the two solutions, $\mathbf{a}_{6}, \mathbf{b}_{1}, \mathbf{b}_{2}$, and $\mathbf{b}_{3}$ define another tetrahedron with known edge lengths. In this case there are two specular possible locations for $\mathbf{b}_{3}$ with respect to the plane defined by $\mathbf{a}_{6}, \mathbf{b}_{1}$ and $\mathbf{b}_{2}$ (Figure 2d). We conclude that, if $\Xi\left(\mathbf{a}_{1}, \mathbf{a}_{2}, \mathbf{a}_{3}, \mathbf{b}_{1}\right), \Xi\left(\mathbf{a}_{4}, \mathbf{a}_{5}, \mathbf{b}_{2}, \mathbf{b}_{3}\right)$, and $\Xi\left(\mathbf{a}_{6}, \mathbf{b}_{1}, \mathbf{b}_{2}, \mathbf{b}_{3}\right)$ are all different from zero, there are 8 possible configurations for the moving platform compatible with a given set of wire lengths. Let us choose one of these solutions and let us infinitesimally modify the wire lengths. This will univocally give a new configuration for the platform. This means that the Jacobian relating the platform and wire velocities is well-defined if, and only if, none of these determinants vanish. In other words, these three determinants fully characterize all the singularities of the tracking system. This characterization is given in terms of the wire lengths, but in most cases we want to give it in terms of 
the configuration of the platform. The solution to this problem curiously comes from the developments in motion planning for polyhedra.

Note that $\Xi\left(\mathbf{p}_{1}, \mathbf{p}_{2}, \mathbf{p}_{3}, \mathbf{p}_{4}\right)$ is zero if, and only if, $\mathbf{p}_{1}, \mathbf{p}_{2}, \mathbf{p}_{3}$, and $\mathbf{p}_{4}$ lie on a plane. In other words, if, and only if, $\left|\begin{array}{ll}\mathbf{p}_{1} & 1 \\ \mathbf{p}_{2} & 1 \\ \mathbf{p}_{3} & 1 \\ \mathbf{p}_{4} & 1\end{array}\right|=0$. As a consequence, when at least one of $\left|\begin{array}{ll}\mathbf{a}_{1} & 1 \\ \mathbf{a}_{2} & 1 \\ \mathbf{a}_{3} & 1 \\ \mathbf{b}_{1} & 1\end{array}\right|,\left|\begin{array}{ll}\mathbf{a}_{4} & 1 \\ \mathbf{a}_{5} & 1 \\ \mathbf{b}_{2} & 1 \\ \mathbf{b}_{3} & 1\end{array}\right|$, or $\left|\begin{array}{ll}\mathbf{a}_{6} & 1 \\ \mathbf{b}_{1} & 1 \\ \mathbf{b}_{2} & 1 \\ \mathbf{b}_{3} & 1\end{array}\right|$ vanishes, the system is in a singular configuration. Now, the problem is to explicitly express these determinants in function of the configuration of the platform $\mathbf{q} \in \Re^{3} \times S O(3)$.

These three determinants, when equated to zero, can be seen as the implicit equations of the C-surfaces in $\Re^{3} \times S O(3)$ associated with each of the three basic contacts between polyhedra (see (Thomas, 1995) for details). In particular, with the face-vertex, edge-edge and vertex-face contact, respectively. The algebraic expressions for these surfaces were first given in (Donald, 1984). They are rather long (particularly the one corresponding to the second determinant), thus requiring a computer algebra system for their manipulation.

Each C-surface, a variety of dimension 5, divides $\Re^{3} \times S O(3)$ into two half-spaces. Then, the three C-surfaces lead to a partition of the configuration space into 8 regions with congruent signs for the 3 determinants. During normal operation, a 3-2-1 wired-based tracking system should work in one of these regions without getting out of it to avoid ambiguities. If one of the above determinants goes below a certain threshold the system should stop under the risk of trespassing a C-surface. The explicit description of these 8 regions is not easy but, if the moving platform is constrained to move in a plane, the configuration space becomes $\Re^{2} \times S O$ and the technique used in (Avnaim et al., 1988) for obtaining such descriptions can be applied. Iterating this for different parallel planes, a discretization can be obtained.

A motion of the platform defines a trajectory in the configuration space, and each point of this trajectory has an associated ellipsoidal uncertainty region. These ellipsoids, as obtained in the previous section, are unbounded if, and only if, the configuration is on a C-surface. In this case, it can be shown that the ellipsoid degenerates into a strip containing the tangent plane to the $\mathrm{C}$-surface at the considered configuration (Figure 3). Thus, even in the case the system is in a singularity, the associated uncertainty remains bounded in the direction orthogonal to the corresponding C-surface. 


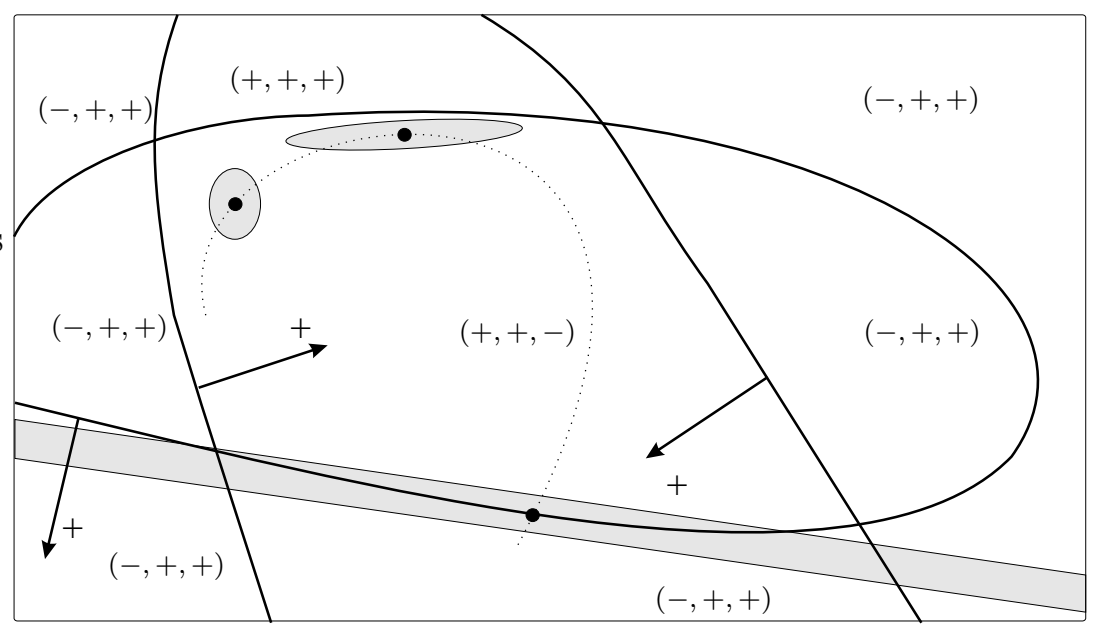

Figure 3. The C-surfaces induce a partition of the configuration space of the moving platform with the same signs for the associated determinants. A motion of the platform corresponds to a trajectory (shown here in dotted line). Each point of this trajectory has an associated uncertainty ellipsoid which degenerates into a strip when trespassing a C-surface.

\section{Conclusions}

An ellipsoidal uncertainty model for wire-based tracking systems with wire length uncertainties and joint clearances has been presented. Using this model, a one-to-one correspondence between kinematic singularities of the tracking system and configurations with unbounded uncertainty arises. Then, although it is possible to characterize the singularities in terms of the geometry of linearly dependent sets of lines, we have presented an ad hoc approach that takes advantage of the 3-2-1 wire configuration. It has also been shown that these singularities, when expressed in the configuration space of the moving platform, can be identified as C-surfaces associated with the three basic contacts between polyhedra. Since the contacts between wires can be formulated in the same terms, this representation of the problem leads to a unifying formalism where the region for safe tracking -i.e. free from singularities and without wire wrapping problems- is a region of the configuration space of the moving platform bounded by a set of C-surfaces. This point, as well as the experimental validation of the presented results concentrates our current efforts. 


\section{Acknowledgements}

This work has been partially supported by the Italian-Spanish Bilateral Cooperation Committee and the Spanish CICYT under contract TIC2000-0696.

\section{References}

F. Avnaim, J.D. Boissonnat and B. Faverjon, A practial motion planning algorithm for polygonal objects amisdst polygonal obstacles, Proc. of the IEEE Int. Conf. on Robotics and Automation, pp. 1656-1661, 1988.

M. Ceccarelli, C. Avila-Carrasco, and E. Ottaviano, Error analysis and experimental tests of CATRASYS (Cassino Tracking System), 2000 Int. Conf. on Industrial Electronics, Control and Instrumentation, IECON 2000, paper SPC11-SP2-4, Nagoya, 2000.

M. Ceccarelli, M.E. Toti, and E. Ottaviano, CATRASYS (Cassino Tracking System): A new measuring system for workspace evaluation of robots, Proc. of the 8th Int. Workshop on Robotics in Alpe-Adria-Danube Region RAAD'99, pp. 19-24, Munich, 1999.

B.R. Donald, Local and Global Techniques for Motion Planning, Master Science Thesis, MIT, 1984.

H. Dörrie, 100 Great Problems of Elementary Mathematics. Their History and Solution, Dover Publications, New York, 1965.

Z. Geng and L.S. Haynes, A 3-2-1 kinematic configuration of a Stewart platform and its application to six degree of freedom pose measurements, Robotics and Computer-Integrated Manufacturing, Vol. 11, No. 1, pp. 23-34, 1994.

J. Hightower and G. Borriello, A survey and taxonomy of location systems for ubiquitous computing, $U W$ CSE-01-08-03, University of Washington, Department of Computer Science and Engineering, 2001.

J.W. Jeong, S.H. Kim, and Y.K. Kwak, Kinematics and workspace analysis of a parallel wire mechanism for measuring a robot pose, Mechanism and Machine Theory, Vol. 34, No. 6, pp. 825-841, 1999.

J.M. McCarthy, Geometric Design of Linkages, Springer-Verlag, New York, 2000.

M. A. Peterson, The geometry of Piero della Francesca, The Mathematical Intelligencer, Vol. 19, No. 3, pp. 33-40, 1997.

H. Pottmann, and J. Wallner, Computational Line Geometry, Springer-Verlag, Berlin, 2001.

L. Ros, A. Sabater, and F. Thomas, An ellipsoidal calculus based on propagation and fusion, IEEE Trans. on Systems, Man and Cybernetics. Part B, Vol. 32, No. 4, to appear, 2002.

S. Tadokoro at al. On the fundamental design of wire driven parallel manipulators with redundancy, In Japan/USA Symposium on Flexible Automation, pp. 151-158, Boston, 1996.

F. Thomas, An approach to the movers' problem that combines oriented matroid theory and algebraic geometry, Proc. of the 1995 IEEE Int. Conf. on Robotics and Automation, Vol. III, pp. 2285-2293, 1995.

J. Zhu and K-L. Ting, Uncertainty analysis of planar and spatial robots with joint clearances, Mechanism and Machine Theory, Vol. 35, pp. 1239-1256, 2000. 